\title{
Characterization of gamma field in the JSI TRIGA reactor
}

\author{
Klemen Ambrožič, Vladimir Radulović, Luka Snoj \\ "Jožef Stefan" Institute, Jamova cesta 39, SI-1000 Ljubljana, Slovenia \\ Adrien Gruel, Mael Le Guillou, Patrick Blaise \\ CEA, DEN, DER, SPEx, Experimental Programs Laboratory \\ Cadarache, F-13108 St. Paul-Lez-Durance, France \\ Christophe Destouches, Loïc Barbot, \\ CEA, DEN, DER, SPEx, Instrumentation Sensors and Dosimetry Laboratory, \\ Cadarache, F-13108 St. Paul-Lez-Durance, France
}

\begin{abstract}
Research reactors such as the "Jožef Stefan" Institute TRIGA reactor have primarily been designed for experimentation and sample irradiation with neutrons. However recent developments in incorporating additional instrumentation for nuclear power plant support and with novel high flux material testing reactor designs, $\gamma$ field characterization has become of great interest for the characterization of the changes in operational parameters of electronic devices and for the evaluation of $\gamma$ heating of MTR's structural materials in a representative reactor $\gamma$ spectrum.
\end{abstract}

In this paper, we present ongoing work on $\gamma$ field characterization both experimentally, by performing $\gamma$ field measurements, and by simulations, using Monte Carlo particle transport codes in conjunction with R2S methodology for delayed $\gamma$ field characterization.

Index Terms-Gamma-rays, Nuclear electronics, Radiation hardening (electronics), Reactor instrumentation, Scintillators.

\section{INTRODUCTION}

The "Jožef Stefan" Institute (JSI) TRIGA reactors is a pool type, $250 \mathrm{~kW}$ reactor with $\mathrm{U} \mathrm{Zr}-\mathrm{H}$ fuel elements arranged in an annular core and equipped with diverse irradiation facilities, which have during its 50 years of operation [1] been used primarily for neutron experimentation such as neutron radiography [2], neutron activation analysis [3], bulk material testing [4], [5], [6] and electronic component radiation hardness studies [7], [8], etc.

The neutron field in irradiation facilities has been thoroughly computationally characterized [9] and experimen- tally validated [10]. Experiments have also been included in the International Reactor Physics Experimental Evaluation Project (IRPhEP) handbook [11], and the reactor has become a reference center for neutron irradiation of detectors for the ATLAS experiment in CERN [12].

Recent increased interest in development of radiation tolerant electronic devices for operation inside Nuclear Power Plant (NPP) containment buildings [13], particle accelerators [14], aerospace applications [15], [16] and for evaluation of structural material $\gamma$ heating in Material testing reactors (MTR) [17], [18] has highlighted the need for utilization of reactor irradiation positions for pure $\gamma$ irradiation.

$\gamma$-rays inside a nuclear reactor can generally be divided into two groups by their mode of creation: prompt $\gamma$ rays, which are produced during reactor operation and are emitted promptly after nucleus interaction, such as fission [19], scattering [20] or radiative capture [21]. On the other hand, delayed $\gamma$-rays are emitted as a consequence of radioactive decay of fission and activation products. To date, evaluations suggest that delayed $\gamma$ field contributes more then $30 \%$ to total $\gamma$ flux during reactor operation [22], [23], and is the only major source of $\gamma$ rays after reactor shutdown.

Characterizing the $\gamma$ field and distinguishing the time dependent (delayed) and independent (prompt) contributions presents several challenges that we aim to overcome. The characterization can roughly be divided into two parts: computational characterization and modeling and experimental validation. Novel techniques for de- 
layed $\gamma$ modeling coupling steady state Monte Carlo particle transport codes with radioactive inventory calculations codes such as the Rigorous-2-Step (R2S) [24] methodology are utilized and implemented, and numerous detector types are used for experimental validation, measuring both integral and differential quantities of interest.

The established framework will serve for routine qualification of the $\gamma$ field in the JSI TRIGA irradiation facilities $\gamma$ field and sample responses to radiation. The method could also be applied later on for qualification of activated material prior to decommissioning of nuclear reactors.

\section{JSI TRIGA REACTOR}

The JSI TRIGA reactor is controlled by 4 control rods and is equipped with numerous irradiation ports, both in-core, placed in the fuel element positions with the exception of the Triangular channel (TriC) which occupies 3 fuel element positions (Fig. 3), and ex-core, with 40 irradiation positions located inside the graphite reflector, which can be rotated into desired position by a rotary carousel. Some of the in-core and carousel irradiation positions are equipped with a fast pneumatic transfer system for sample irradiation. There are also 3 larger horizontal irradiation positions with inner radius of $15 \mathrm{~cm}$. Two of them extending radially outwards from the reactor core, Radial Piercing Port (RPP) piercing the graphite reflector and Radial Beam Port (RBP) extending outwards from the graphite reflector. The Tangential Channel (TanC) horizontal irradiation position passes the reactor core tangentially. The in-core irradiation

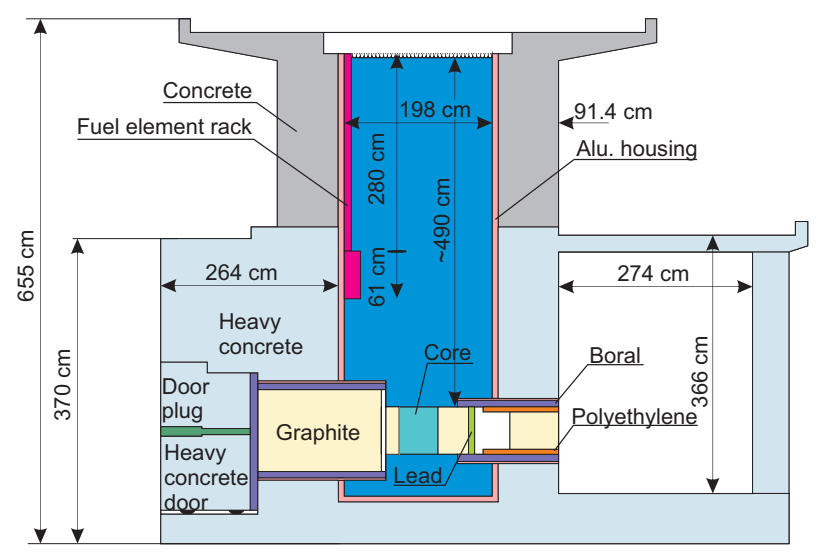

Fig. 1. JSI TRIGA reactor side-cut view schematic.

positions have graphite or aluminum spacers at the bottom, which define the sample height in relation to the fuel elements to fuel element center-height. Samples are usually lowered into positions inside aluminum or polyethylene capsules.

Fuel elements can also be extracted from the reactor

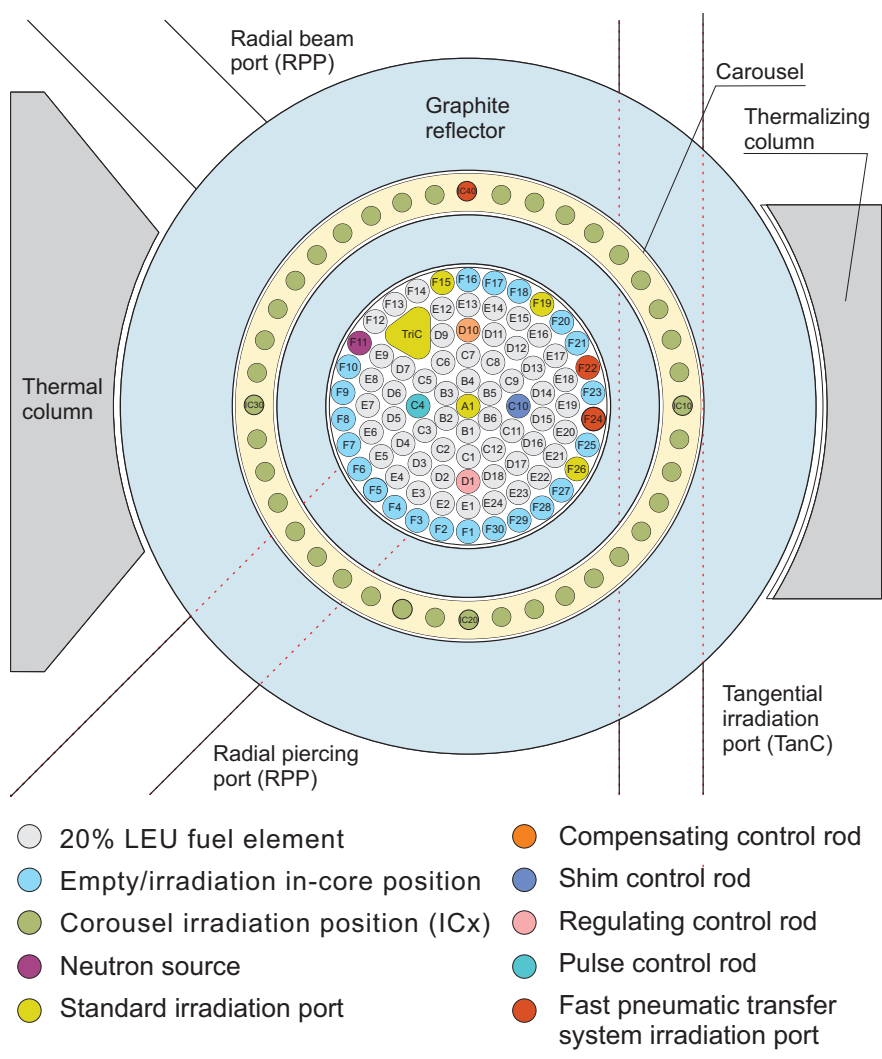

Fig. 2. JSI TRIGA reactor core schematic with core configuration no. 189.

core and relocated to the fuel element rack located at the edge of the reactor rank (Fig.1), which can hold up to 6 fuel elements. Since fuel elements are highly radioactive after reactor operation, they can be used as a viable pure $\gamma$-ray source. For pure $\gamma$ irradiation with such an arrangement, an additional irradiation facility in form or an aluminum box with inner dimensions of $30 \mathrm{~cm} \times 20 \mathrm{~cm} \times 20 \mathrm{~cm}$, submerged to close proximity of the fuel elements, with a cable guide tube leading to the reactor pool surface has been constructed especially for $\gamma$ irradiation of larger electronic components and assemblies (Fig. 4).

The existing irradiation positions were primarily used for neutron irradiation of samples, and have only recently been utilized for $\gamma$ irradiation after reactor shutdown. Although $\gamma$ dose-rates after reactor shutdown exceed $1 \times 10^{4} \mathrm{~Gy} \mathrm{~h}^{-1}$, the $\gamma$ spectrum is time dependent and difficult to measure with a spectrometer due to the high dose-rate, further investigation in characterizing the $\gamma$ 


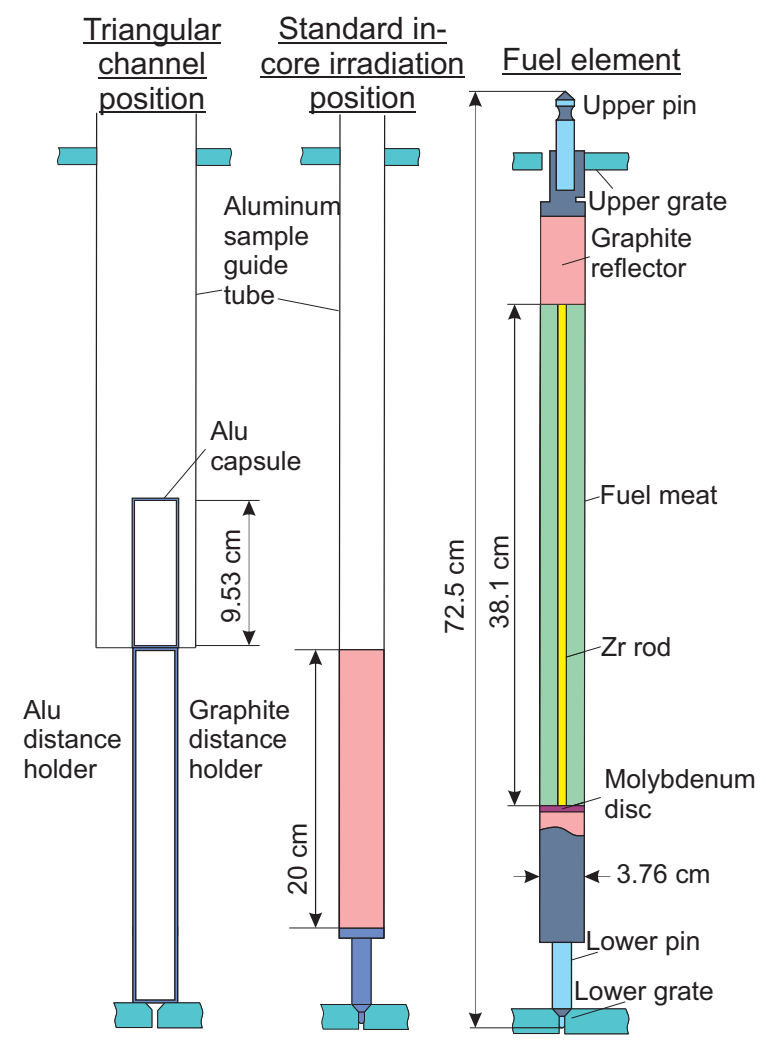

Fig. 3. JSI TRIGA fuel elements and in-core irradiation position schematics.

field is required both computationally and by use of different detectors.

\section{GAMMA FIELD MEASUREMENTS}

The measurements of the $\gamma$ field inside a strong mixed neutron- $\gamma$ field presents several difficulties such as discrimination between neutron and $\gamma$ contributions, and further, discriminating between prompt and delayed $\gamma$ field, where the latter is also dependent on reactor operational history. In case of the JSI TRIGA, where the reactor power ranges from $10 \mathrm{~W}$ to $2.5 \times 10^{5} \mathrm{~W}$, and taking into account background radiation, which is in order of few $\mathrm{Gy} \mathrm{h}^{-1}$, detectors with measurement ranges spanning at least 6 orders of magnitude must be used. Ionization and fission chambers, radiation sensing field effect transistors (RadFETs) [25] and thermoluminescent dosimeters (TLD) using novel readout and annealing techniques [26] have proven to be prime candidates for $\gamma$ field measurements inside a reactor.

Discrimination between neutron and $\gamma$ contributions is usually performed by using similar detectors, which have different sensitivities to different particle types, as is the case with fission [27] and ionization chambers [28],

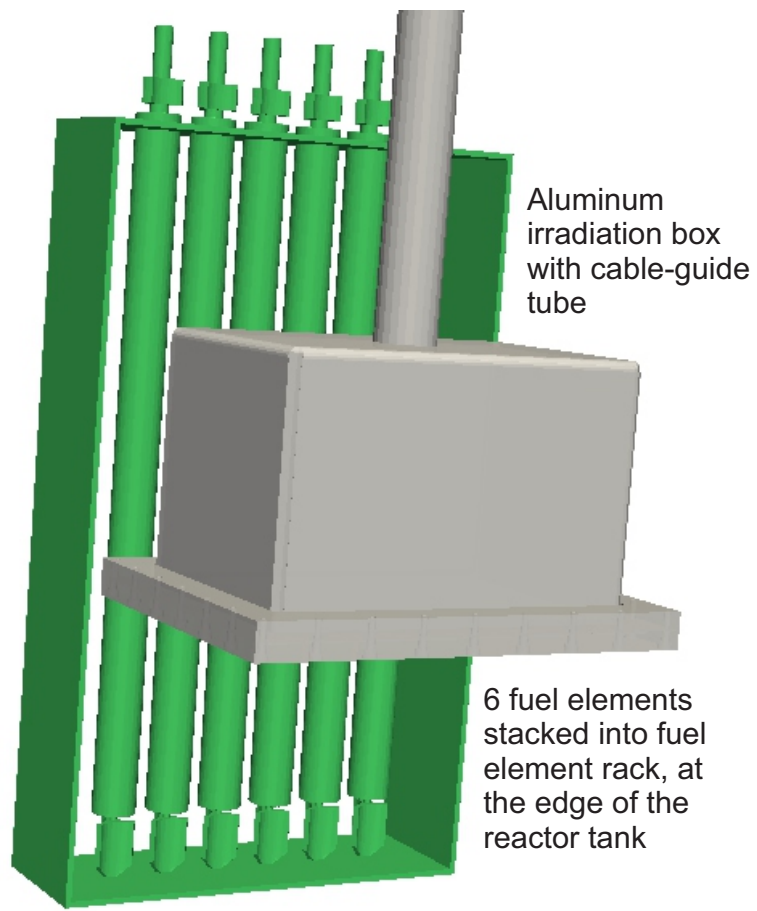

Fig. 4. Schematic of 6 fuel elements stacked in a rack, with Al. irradiation box at close proximity.

[29], as well as for TLD detectors, where some can be loaded with natural lithium and others with enriched ${ }^{7} \mathrm{Li}$. However, qualifying the RadFETs and their temperature dependence [30] is still in progress, and solutions such as different oxide layer thickness and encapsulation of detectors with a neutron filter such as $\mathrm{Cd}$, or by increasing $\gamma$ production by applying a thin layer of $\mathrm{B}$ on the RadFET surface [31] are under investigation.

Radiation detectors measure radiation by charge collection (IC) or trapping (TLD, OSLD), which is released by radiation interaction with matter, so charged particle equilibrium (CPE) must be established in order for the detector to measure the correct quantity [32]. The measured quantity must therefore previously be qualified in a stable $\gamma$ source, such as ${ }^{137} \mathrm{Cs},{ }^{60} \mathrm{Co}$, X-ray and others, preferably high energy sources. CPE is achieved by encapsulating the sensitive part of the detector with an equilibrator part of sufficient thickness [33]. The material of the equilibrator dictates the detector response function, since the actual dose on the equilibrator material is measured. $\gamma$ ray energies inside a reactor reaching up to $10 \mathrm{MeV}$ and further must also be considered. Although the average $\gamma$ energy is in order of an $\mathrm{MeV}$ and is comparable with ${ }^{60} \mathrm{Co}$ spectrum, pair production becomes the dominant interaction process at higher energies instead 
of Compton effect [32], and therefore larger equilibrator thicknesses are required.

While these detectors can be used for a range of reactor powers, they still only measure integral responses, and give no information on incident $\gamma$ energy. To this end a novel, miniature, scintillator crystal based spectrometer is under development. Currently, optimizations on scintillation crystal size and readout techniques are being performed, in order not to oversaturate the crystal and read piled-up pulses, but still have a crystal large enough, for $\gamma$-rays to deposit all of their energy inside the scintillation crystal. Techniques on resolving pile-up pulses [34] somewhat relax these constraints.

A joint JSI and French Atomic Energy and Alternative Energies Commission (CEA) $\gamma$ measurement campaign has already been performed at the JSI TRIGA reactor, where all of the above mentioned detectors (or development versions for detectors under development ) were utilized for $\gamma$-ray measurements. A compact core configuration has been established in order to have a homogeneous neutron field, and reactor operation was planned beforehand, gradually increasing the reactor power from zero power, with extracted neutron source, to establish baseline background signal, to full power in a series of runs, increasing the power exponentially. Special detector holders were constructed for positioning the detectors in centers of their respective irradiation positions, in order to minimize experimental uncertainty. Two fission chambers were also inserted symmetrically to the reactor core center for additional reactor power reading and determining the flux redistribution due to control rod positions. One of the ionization chambers was positioned in a fixed position, while others were used for axial and radial $\gamma$ profile measurements. A RadFET detector and scintillation spectrometer were also utilized, and were lowered in the carousel positions, in order not to oversaturate them or to minimize impact on the detector. During the operations, several sets of TLDs in aluminum equilibrators provided by the CEA were enclosed inside a polyethylene capsule and lowered into 3 different positions: in-core Central channel (A1) and F26 and carousel IC40 position at different reactor powers.

On-line measurements during reactor transients such as power increase (Fig.5) and reactor scram (Fig.6) are of special interest, since delayed $\gamma$ flux time dependency can directly be extracted, by appropriate IC and FC detector signal weighting and subtraction.

These experiments will be repeated again, in a similar fashion, aiming to replicate the conditions of the

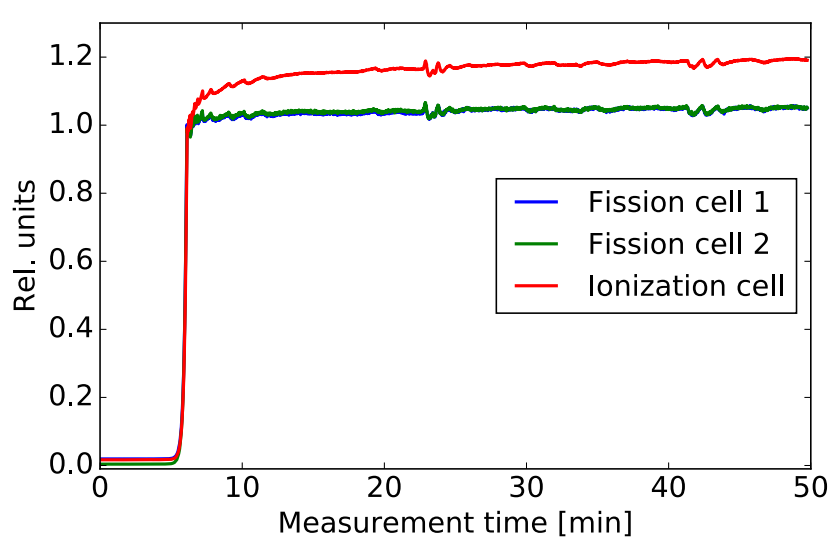

Fig. 5. Fission and ionization cell signal, normalized to value after reactor start-up to $4 \mathrm{~kW}$. Fission chamber signals overlap, while ionization chamber signal increases with time due to neutron activation and delayed $\gamma$-rays.

previous experimental campaign, although using another set of TLD detectors without equilibrators, provided by Institute of Nuclear Physics, Polish Academy of Sciences (IJF-PAN), different type of RadFET detectors and an improved version of the scintillation spectrometer, with fission and ionization cells being the same. The aim is, to reproduce these measurements several times, with a large number of detectors in order to minimize measurement uncertainty. The obtained experimental results will serve for validation of the delayed $\gamma$ computational scheme.

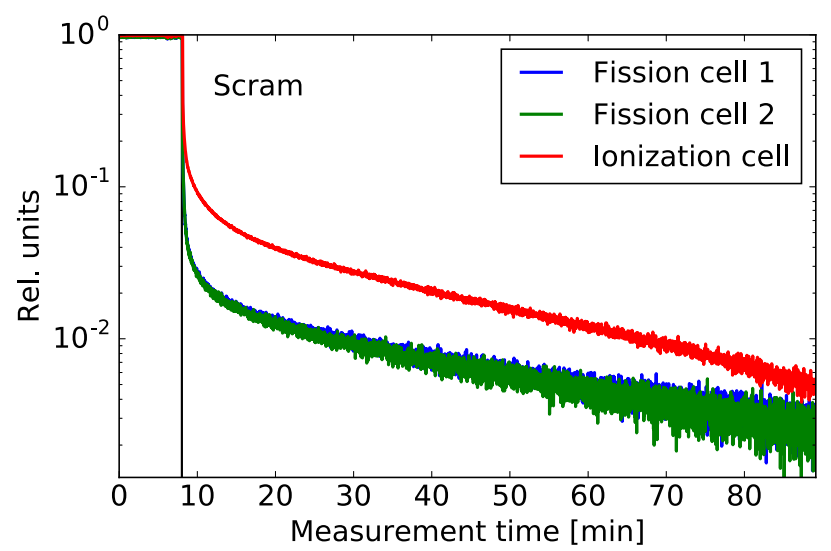

Fig. 6. Fission and ionization cell signal, after scram from steadystate $200 \mathrm{~W}$, showing the delayed $\gamma$ emitters decaying, and contributing to ionization chamber signal. Online measurements for long periods after a scram can provide valuable information about delayed $\gamma$, even for longer-lived activation products. 


\section{Computational modeling}

Computational modeling of the $\gamma$ field inside a nuclear reactor needs to be able to couple transport of neutrons and $\gamma$-rays for kerma approximation. We used the Monte Carlo particle transport code MCNP v6.1 (MCNP) [35] and ENDF/B-VII.0 [36] for prompt $\gamma$ irradiation. The reactor computational model is based on an experimental benchmark computational model [37], with added details, such as fuel holding pins, additional irradiation positions, reactor instrumentation to most accurately reflect the real state of the reactor. Since MCNP only enables steady state eigenvalue calculations, only the prompt part of the $\gamma$ field is calculated directly by MCNP. The $\gamma$ flux and anbient dose equivalents, derived by the use of ICRP-21 [38] and ANSI-ANS6.1.1 ambient dose equivalent factors were calculated throughout the reactor. In the present work, core loading pattern 189 (Fig.2) was simulated, with transient and safety control rod fully withdrawn, regulating rod at position 382 and shim rod in position 350 and TriC inserted into the core. Results in Fig. 7 shows only prompt $\gamma$ contribution, with measurements suggesting an increase $\approx 30 \%$, once the delayed $\gamma$ are accounted for. For accurate estimation of the delayed $\gamma$ contribution, an R2S methodology Python [39] script was developed, coupling MCNP and FISPACT-II [40] radioactive inventory calculation code, which has only been applied to fusion applications [41], [42] and has to our knowledge never been applied to a fission reactor before. The model geometry is divided by a 3D mesh, where the neutron flux and spectra and

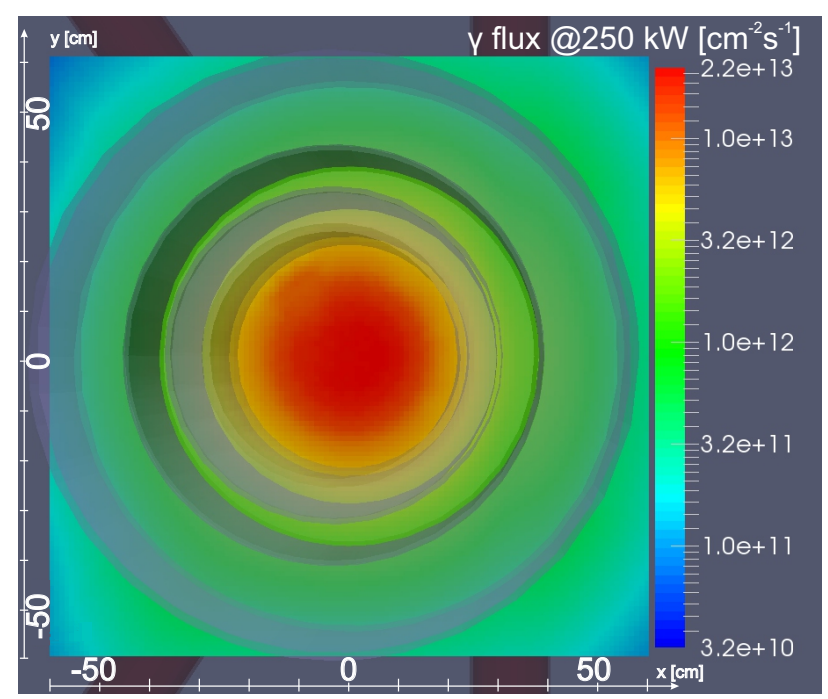

Fig. 7. Prompt $\gamma$ flux in the JSI TRIGA core at fuel element center height @250kW. homogenized material compositions are calculated in each mesh voxel. Inventory calculations are performed using these data and reactor operation parameters, such as reactor power, operation duration and cool-down to calculate radioactive isotope inventory at each time of interest. Delayed particle spectra are calculated for each voxel of the mesh, and secondary particle transport calculations performed with MCNP and a source routine developed at KIT [43].

To accurately reproduce the measurements obtained experimentally, one must consider renormalization of computational results to the reactor power [44], as well as flux redistribution [45] due to control rod insertion and its effects on reactor power readout instrumentation. In addition, secondary particles other then $\gamma$-rays should also be transported, as they may produce additional $\gamma$ rays due to their high energy. Special care must also be taken for energy and charge deposition inside the detectors and electron and positron transport must be considered as well, since the detector size is usually smaller then the characteristic dimension required to achieve CPE.

Simulating different particle types, especially charged particles, required in order to accurately reproduce detector responses and additional delayed $\gamma$ generation, is however computationally very intensive, and special care should be taken in order to optimize accuracy vs. computational time.

\section{Conclusions}

With the development of novel, high flux reactors and the need for radiation tolerant electronics for nuclear and aerospace technology, $\gamma$ field effects studies on reactor component heating and changes in operational parameters of electronic components and assemblies, $\gamma$ field characterization of JSI TRIGA irradiation facilities will enable further studies and development in that area. With a thoroughly characterized $\gamma$ field, sample characteristics changes will be more accurately qualified.

An R2S methodology code, coupling MCNP and FISPACT-II codes for delayed $\gamma$ field calculations, has been developed an with addition of prompt $\gamma$ field calculations performed by the MCNP code, a generalized framework for reactor $\gamma$ field calculations will be established. In addition, results from experimental $\gamma$ measurement campaigns at the JSI TRIGA reactor will serve for code validation and improvement, as well as further development of novel detector types, such as before mentioned $\gamma$ spectrometer. Due to the novelty of R2S methodology use in the irradiation facilities of a 
fission reactor, special care should be taken to obtain experimental results of benchmark quality, on which other R2S methodology codes could also be tested and verified.

The established computational framework will not only serve for qualification of sample responses under $\gamma$ irradiation, but could also serve for qualification of radioactive inventory in reactor structural materials, which will become of great interest after reactor decommissioning and long-term storage, leading to waste storage space optimization and minimizing the associated cost.

\section{REFERENCES}

[1] B. Smodiš and L. Snoj, "Utilization and Application of the Slovenian TRIGA Reactor," "Jožef Stefan" Institute, Ljubljana, Slovenia, Tech. Rep., 2011. [Online]. Available: http://www-pub.iaea.org/MTCD/Publications/PDF/ P1575_CD_web/datasets/papers/A29 $\ \% 20$ Smodis.pdf

[2] J. Rant, Z. Milič, J. Istenič, T. Knific, I. Lengar, and A. Rant, "Neutron radiography examination of objects belonging to the cultural heritage," Applied Radiation and Isotopes, vol. 64, no. 1 , pp. 7 - 12, 2006. [Online]. Available: http://www. sciencedirect.com/science/article/pii/S096980430500206X

[3] A. R. Byrne, "Neutron activation analysis using TRIGA," "Jožef Stefan" Institute, Ljubljana, Slovenia, Italy, Tech. Rep., 1972, iNIS-IT-09N0001.

[4] E. Huseynov, A. Garibov, and R. Mehdiyeva, "Effect of neutron flux, temperature and frequency on the permittivity of nanocrystalline silica," International Journal of Modern Physics $B$, vol. 28 , no. 30, p. 1450213, 2014.

[5] —, "Influence of neutron irradiation and temperature on the electric conductivity of $\mathrm{SiO} 2$ nanoparticles," Journal of Electrostatics, vol. 74, pp. 73 - 78, 2015. [Online]. Available: http://www.sciencedirect.com/ science/article/pii/S0304388615000029

[6] — " "TEM and SEM study of nano $\mathrm{SiO} 2$ particles exposed to influence of neutron flux," Journal of Materials Research and Technology, vol. 5, no. 3, pp. $213-$ 218, 2016. [Online]. Available: http://www.sciencedirect.com/ science/article/pii/S2238785415001106

[7] I. Mandić, V. Cindro, A. Gorišek, G. Kramberger, and M. Mikuž, "Online integrating radiation monitoring system for the ATLAS detector at the Large Hadron Collider," IEEE Transactions on Nuclear Science, vol. 54, no. 4, pp. 1143-1150, Aug 2007.

[8] G. Kramberger, V. Cindro, I. Mandić, M. Mikuž, and M. Zavrtanik, "Determination of detrapping times in semiconductor detectors," Journal of Instrumentation, vol. 7, no. 04, p. P04006, 2012.

[9] L. Snoj, G. Žerovnik, and A. Trkov, "Computational analysis of irradiation facilities at the JSI TRIGA reactor," Applied Radiation and Isotopes, vol. 70, no. 3, pp. 483 488, 2012. [Online]. Available: http://www.sciencedirect.com/ science/article/pii/S0969804311005963

[10] L. Barbot, C. Domergue, S. Braud, C. Destouches, J. F. Villard, L. Snoj, Štancar Ž., V. Radulović, and A. Trkov, "Neutron field characterization of irradiation locations applied to the slovenian TRIGA reactor," in Advancements in Nuclear Instrumentation Measurement Methods and their Applications (ANIMMA), 2013 3rd International Conference on, June 2013, pp. 1-5.
[11] E. Sartori and J. Blair Briggs, "IRPhEP-handbook, International Handbook of Evaluated Reactor Physics Benchmark Experiments," Nuclear Energy Agency of the OECD (NEA), Mar 2008.

[12] L. Snoj and B. Smodiš, "45 Years of TRIGA Mark II in Slovenia," in Proc. International Conference Nuclear Energy for New Europe 2011, 2011, article no. 401.

[13] A. F. Fernandez, A. I. Gusarov, B. Brichard, S. Bodart, K. Lammens, F. Berghmans, M. Decreton, P. Megret, M. Blondel, and A. Delchambre, "Temperature monitoring of nuclear reactor cores with multiplexed fiber bragg grating sensors," Optical Engineering, vol. 41, no. 6, pp. 1246-1254, 2002. [Online]. Available: http://dx.doi.org/10.1117/1.1475739

[14] A. Serrano, J. Andrés, N. P. Barradas, F. M. Cardeira, J. CasasCubillos, A. C. Fernandes, F. Peláez, P. Gomes, I. C. Gonçalves, A. Hernández Cachero et al., "Radiation tests on commercial instrumentation amplifiers, analog switches \& DAC's," in Proceedings of the 8th Conference on Electronics for LHC Experiments. CERN Publishing Service, 2001, pp. 117-121.

[15] R. C. Lacoe, J. V. Osborn, R. Koga, S. Brown, and D. C. Mayer, "Application of hardness-by-design methodology to radiationtolerant ASIC technologies," IEEE Transactions on Nuclear Science, vol. 47, no. 6, pp. 2334-2341, Dec 2000.

[16] S. Duzellier, "Radiation effects on electronic devices in space," Aerospace Science and Technology, vol. 9, no. 1, pp. $93-$ 99, 2005. [Online]. Available: http://www.sciencedirect.com/ science/article/pii/S1270963804001129

[17] H. Amharrak, J. D. Salvo, A. Lyoussi, A. Roche, M. MassonFauchier, J. C. Bosq, and M. Carette, "Analysis and recent advances in gamma heating measurements in MINERVE facility by using TLD and OSLD techniques," in 2011 2nd International Conference on Advancements in Nuclear Instrumentation, Measurement Methods and their Applications, June 2011, pp. 1-9.

[18] M. Lemaire, C. Vaglio-Gaudard, A. Lyoussi, and C. ReynardCarette, "For a better estimation of gamma heating in experimental reactors and devices: Stakes and work plan from calculation methods to nuclear data," in 2013 3rd International Conference on Advancements in Nuclear Instrumentation, Measurement Methods and their Applications (ANIMMA), June 2013, pp. 1-6.

[19] F. Pleasonton, R. L. Ferguson, and H. Schmitt, "Prompt gamma rays emitted in the thermal-neutron-induced fission of u 235," Physical Review C, vol. 6, no. 3, p. 1023, 1972.

[20] R. B. Day, "Gamma rays from neutron inelastic scattering," Phys. Rev., vol. 102, pp. 767-787, May 1956. [Online]. Available: https://link.aps.org/doi/10.1103/PhysRev.102.767

[21] M. Lone, R. Leavitt, and D. Harrison, "Prompt gamma rays from thermal-neutron capture," Atomic Data and Nuclear Data Tables, vol. 26, no. 6, pp. 511 559, 1981. [Online]. Available: http://www.sciencedirect.com/ science/article/pii/0092640X8190005X

[22] D. Fourmentel, V. Radulović, L. Barbot, J. F. Villard, G. Žerovnik, L. Snoj, M. Tarchalski, K. Pytel, and F. Malouch, "Delayed Gamma Measurements in Different Nuclear Research Reactors Bringing Out the Importance of Their Contribution in Gamma Flux Calculations," IEEE Transactions on Nuclear Science, vol. 63, no. 6, pp. 2875-2879, Dec 2016.

[23] V. Radulović, D. Fourmentel, L. Barbot, J.-F. Villard, T. Kaiba, Ž. Gašper, and L. Snoj, "Measurements of miniature ionization chamber currents in the JSI TRIGA Mark II reactor demonstrate the importance of the delayed contribution to the photon field in nuclear reactors," Nuclear Instruments and Methods in Physics 
Research Section A: Accelerators, Spectrometers, Detectors and Associated Equipment, vol. 804, pp. 149-154, 2015.

[24] A. Davis, "Radiation Shielding of Fusion Systems," Ph.D. dissertation, University of Birmingham, 2010.

[25] F. Ravotti, "Development and characterization of radiation monitoring sensors for high energy physics experiments of the CERN LHC accelerator,' Ph.D. dissertation, L'UNIVERSITE MOTPELLIER II, November 2006.

[26] B. Obryk, P. Batistoni, S. Conroy, B. D. Syme, S. Popovichev, I. E. Stamatelatos, T. Vasilopoulou, and P. Bilski, "Thermoluminescence measurements of neutron streaming through JET Torus Hall ducts," Fusion Eng. Des., vol. 89, no. 9-10, pp. 2235-2240, 2013.

[27] S. Normand, P. Delacour, B. Lescop, L. Laribiere, T. Nguyen, C. Blandin, and J.-C. Trama, "A new small fission chamber for in core and wide range neutron flux measurement," CEA, Tech. Rep., 2006.

[28] U. Chica, M. Anguiano, M. Vilches, and A. M. Lallena, "Quality indexes based on water measurements for low and medium energy X-ray beams: A theoretical study with PENELOPE," Medical Physics, vol. 41, no. 1, pp. 012 101-n/a, 2014, 012101. [Online]. Available: http://dx.doi.org/10.1118/1. 4836556

[29] D. Fourmentel, P. Filliatre, J. Villard, A. Lyoussi, C. ReynardCarette, and H. Carcreff, "Measurement of photon flux with a miniature gas ionization chamber in a material testing reactor," Nuclear Instruments and Methods in Physics Research Section A: Accelerators, Spectrometers, Detectors and Associated Equipment, vol. 724, pp. 76 82, 2013. [Online]. Available: http://www.sciencedirect.com/ science/article/pii/S0168900213005214

[30] J. Hofman, A. Holmes-Siedle, R. Sharp, and J. Haze, "A method for in-situ, total ionising dose measurement of temperature coefficients of semiconductor device parameters," IEEE Transactions on Nuclear Science, vol. 62, no. 6, pp. 2525-2531, Dec 2015.

[31] J. K. Shultis and D. S. McGregor, "Efficiencies of coated and perforated semiconductor neutron detectors," IEEE Transactions on Nuclear Science, vol. 53, no. 3, pp. 1659-1665, June 2006.

[32] F. H. Attix, Introduction to radiological physics and radiation dosimetry. John Wiley \& Sons, 2008.

[33] H. Amharrak, J. D. Salvo, A. Lyoussi, P. Blaise, M. Carette, A. Roche, M. Masson-Fauchier, A. Pepino, and C. ReynardCarette, "Development and optimization of nuclear heating measurement techniques in zero power experimental reactors," IEEE Transactions on Nuclear Science, vol. 61, no. 5, pp. 25152526, Oct 2014.

[34] M. Miklavec, B. Löher, D. Savran, R. Novak, S. Širca, and M. Vencelj, "Pile-up correction techniques for real-time dosimetry in photon radiotherapy," in Nuclear Science Symposium and Medical Imaging Conference (NSS/MIC), 2012 IEEE. IEEE, 2012, pp. 3880-3882.

[35] J. T. Goorley, M. R. James, T. E. Booth, F. B. Brown, J. S. Bull, L. J. Cox, J. W. J. Durkee, J. S. Elson, M. L. Fensin, R. A. I. Forster, and et al., Initial MCNP6 Release Overview - MCNP6 version 1.0. Los Alamos National Laboratory (LANL), Jun 2013, Report number: LANL Report LA-UR-13-22934. [Online]. Available: http://www.osti.gov/scitech/servlets/purl/1086758

[36] M. Chadwick, P. Obložinskỳ, M. Herman, N. Greene, R. McKnight, D. Smith, P. Young, R. MacFarlane, G. Hale, S. Frankle et al., "ENDF/B-VII. 0: Next Generation Evaluated Nuclear
Data Library for Nuclear Science and Technology," Nuclear data sheets, vol. 107, no. 12, pp. 2931-3060, 2006.

[37] R. Jeraj and M. Ravnik, "Reactor U(20)-Zirconium Hydride Fuel Rods In Water With Graphite Reflector, International Handbook of Evaluated Criticality Safety Benchmark Experiments," NEA/NSC/DOC/(95) 03/III, Tech. Rep., 1999.

[38] J. Hale, "Data for Protection Against Ionizing Radiation from External Sources: Supplement to ICRP Publication 15. ICRP Publication 21. By The International Commission on Radiological Protection. paper, pp.100, Elmsford, N.Y., Pergamon Press, 1973." Radiology, vol. 111, no. 3, pp. 716-716, jun 1974. [Online]. Available: http://dx.doi.org/10. $1148 / 111.3 .716 b$

[39] G. van Rossum and F. L. Drake, The Python Language Reference Manual. Network Theory Ltd., 2011.

[40] J.-C. Sublet C, J. W. Eastwood, J. G. Morgan, M. Fleming, and M. R. Gilbert, The FISPACT-II User Manual, issue 7 ed., UK Atomic Energy Authority, Culham Science Centre, Abingdon, Oxfordshire, OX14 3DBr, 92015.

[41] P. Sauvan, J. P. Cataln, F. Ogando, R. Jurez, and J. Sanz, "Development of the R2SUNED Code System for Shutdown Dose Rate Calculations," IEEE Transactions on Nuclear Science, vol. 63, no. 1, pp. 375-384, Feb 2016.

[42] P. Batistoni, M. Angelone, L. Petrizzi, and M. Pillon, "Benchmark experiment for the validation of shut down activation and dose rate in a fusion device," Journal of Nuclear Science and Technology, vol. 39, no. sup2, pp. 974-977, 2002.

[43] D. Leichtle and E. Relson. (2013) Source rutine for cartesian steady state gamma source. Karsruhe Institue of Technology. [Online]. Available: https://github.com/svalinn/ r2s-act/blob/master/mcnp_source/source_gamma.F90

[44] G. Žerovnik, M. Podvratnik, and L. Snoj, "On normalization of fluxes and reaction rates in MCNP criticality calculations," Annals of Nuclear Energy, vol. 63, pp. 126 - 128, 2014. [Online]. Available: http://www.sciencedirect.com/ science/article/pii/S0306454913004003

[45] Ž. Štancar, L. Snoj, and L. Barbot, "Analysis of the TRIGA Mark II Research Reactor Ex-Core Detector Response," in 2015 24th International Conference Nuclear Energy for New Europe. Društvo jedrskih strokovnjakov, September 2015, pp. 31-32. 